\title{
Experiências Pedagógicas no Desenvolvimento e Adequação Didática de Protótipos para Práticas Multidisciplinares em Cursos de Engenharia
}

DOI: 10.37702/2175-957X.COBENGE.2021.3394

Rômulo Lira Milhomem - romulo.milhomem@ifsc.edu.br

IFSC

89813-000 3450D

89813-000 - Chapecó - SC

Leonardo José Fiori - leonardo.jf@aluno.ifsc.edu.br

IFSC

R. Campinas 84

89805-697 - CHAPECO - SC

Resumo: O desenvolvimento de protótipos didáticos é uma prática de ensino-aprendizagem consolidada no sentido de contribuir para a formação dos estudantes envolvidos, visto que é uma ferramenta que favorece a integração entre teoria e prática, que aponta a um processo formativo voltado ao saber fazer, introduzindo os estudantes envolvidos ao mundo do trabalho que serão inseridos. Assim, neste trabalho é ilustrada uma experiência do Campus Chapecó, do Instituto Federal de Santa Catarina, no desenvolvimento e adequação de protótipos didáticos para práticas multidisciplinares no curso de Engenharia de Controle e Automação. São relatados os resultados teóricos e práticos e seus impactos na pesquisa e ensino da comunidade acadêmica em que se insere, sob a ótica docente e discente.

Palavras-chave: Protótipos didáticos, Engenharia de Controle e Automação, Educação em Engenharia. 


\section{Experiências Pedagógicas no Desenvolvimento e Adequação Didática de Protótipos para Práticas Multidisciplinares em Cursos de Engenharia}

\section{INTRODUÇÃO}

A didática pode ser definida como um conjunto de atividades organizadas pelo docente, visando o favorecimento da construção do conhecimento pelo estudante (FIORE FERRARI; LEYMONIE SAENZ, 2018), que pode compreender três dimensões distintas em seu campo de atuação, sendo: o estudante, os culturais e o docente. O estudo dessas dimensões pode ajudar a conhecer melhor os processos de aprendizagem dos estudantes e de ensino dos docentes (ÁLVARES, 2006). Quando se observa os aspectos dessas três dimensões em cursos de graduação das áreas tecnológicas, verificam-se deficiências na formação do universitário devido a práticas pedagógicas que tangenciam a aplicação/correlação de conceitos a experimentos práticos (ÁLVARES, 2006).

Nesse contexto, a fabricação e utilização de bancadas/protótipos didáticos são atividades consideradas como boas opções para desenvolvimento da aprendizagem, uma vez que integram esforços de discentes e docentes, visando correlacionar atividades práticas a conceitos teóricos vistos em sala de aula. Além disso, a construção de um material de apoio didático-pedagógico busca aumentar a integração entre discentes e docentes, contribuindo diretamente para o desenvolvimento das habilidades cognitivas do aluno por meio da integração de práticas e conceitos ainda que não repassados em sua totalidade (OLIVEIRA; TOZZI; LODER, 2014).

Assim, busca-se apresentar, no presente trabalho, as características de construção e adequação didática do sistema desenvolvido em FUZINATTO E MILHOMEM (2018), visando simular um processo de controle de velocidade, onde os estudantes podem observar e estudar a dinâmica deste fenômeno físico, cuja aplicação é presente em vários processos industriais. Os protótipos desenvolvidos têm a finalidade de auxiliar discentes e docentes no processo de ensino/aprendizagem na área da engenharia de controle e automação, elétrica e eletrônica, caracterizando a multidisciplinaridade da aplicação do processo. Tangenciando os aspectos técnicos, busca-se apresentar a experiência pedagógica do discente produtor do projeto.

O protótipo realiza a medida da velocidade angular do processo, aplicando diferentes tipos de sinais de entrada e de perturbação, assim como permite controle de velocidade angular do motor. Dessa maneira, o sistema permite uma interação entre o estudante e a bancada, utilizando-se de uma interface, tanto para extração de dados para a modelagem matemática, como para alterar parâmetros de controle. Adicionalmente, a adequação do sistema de FUZINATTO E MILHOMEM (2018), também está relacionada ao desenvolvimento de um projeto de sistema que possa ser facilmente replicado, utilizando peças e componentes comumente comercializados ou que possam ser fabricados em impressoras 3D, caracterizando a modularidade do sistema e a facilidade de manutenção.

Nesse sentido, na Seção 2, serão apresentados os aspectos construtivos das bancadas desenvolvidas nesse trabalho, assim como a metodologia adotada para a construção do sistema. Na Seção 3, apresentam-se os resultados práticos e de aplicação do sistema proposto. E, por fim, na Seção 4 as conclusões do trabalho são apresentadas, observando que parte do projeto foi realizado com docente e discente atuando de forma remota, o que trouxe um desafio maior ao trabalho. Além disso, é realizada uma projeção para o futuro das bancadas e sua contribuição para o ensino de engenharia, principalmente no campo da engenharia de controle e automação. 


\section{ASPECTOS CONSTRUTIVOS E METODOLÓGICOS DOS PROTÓTIPOS}

A fabricação de bancadas/protótipos didáticos apontam à um processo formativo voltado ao saber fazer, introduzindo o estudante produtor ao mundo do trabalho, tendo a organização de suas atividades realizada a partir de uma metodologia de projeto de produto descrita por ROSENFELD E FORCELINI (2006), comumente utilizada na vida profissional do futuro engenheiro. Essa prática tem sido empregada no curso superior de Engenharia de Controle e Automação (ECA) do IFSC, Câmpus Chapecó, em Projetos Integradores ou de Pesquisa Aplicada, como estratégia pedagógica de interdisciplinaridade, envolvendo conceitos de diferentes disciplinas em prol do desenvolvimento de um processo/produto de automação. Neste caso, emprega-se uma metodologia de desenvolvimento de projeto de produto para planejamento das etapas de construção do processo/produto proposto, a partir da aplicação de uma metodologia ativa de Aprendizagem Baseada em Projetos (PBL - do inglês Project Based Learning) para sintetizar os conhecimentos envolvidos no projeto pelo discente (BENDER, 2014).

Assim, optou-se pela utilização um sistema de controle de velocidade em motores de corrente contínua, por sua simplicidade e facilidade de compreensão para construção e aplicação da ferramenta didática, entendendo-se que o conhecimento se dá de forma gradativa e que o sistema proposto pode introduzir o discente a conceitos iniciais de engenharia. De igual forma, a fim de contribuir com o aspecto didático, é imprescindível que os protótipos construídos forneçam, de forma simples e intuitiva, dados ao usuário, como gráficos, sinais para análise e índices de desempenho, facilitando a visualização de parâmetros do sistema e sua influência nas características de controle, a partir da aplicação de testes e demonstrações. Além disso, os protótipos desenvolvidos devem ser modulares, leves e de fácil transporte para que possam ser utilizados em diferentes campos de cursos de engenharia de controle e áreas afins, como a eletrônica, circuitos elétricos e programação, dando destaque a usabilidade das ferramentas físicas e de software e o fornecimento de um manual de utilização e de um material de apoio didático-pedagógico.

Na sequência, serão apresentadas as características construtivas relacionadas ao desenvolvimento do projeto detalhado e do protótipo, que é parte da metodologia de projeto de produtos apresentada por ROSENFELD E FORCELINI (2006). Nesse sentido, os protótipos desenvolvidos foram construídos utilizando materiais disponíveis nos laboratórios, impressão 3D e componentes comumente utilizados para prototipagem. Todos os sistemas são funcionais, e podem ser usados em disciplinas onde se trabalham programação de sistemas embarcados, projetos de controle, análise de sinais e sistemas, e instrumentação.

$\mathrm{Na}$ Erro! Fonte de referência não encontrada.a pode-se observar os componentes de uma unidade produzida. Cada bancada possui um microcontrolador (1), sensores ópticos convertidos à encoders (2) utilizando um disco perfurado (3), chaves para acionamento da perturbação ao sistema (4), um motor elétrico de corrente contínua (5) e a ponte $\mathrm{H}$ utilizada para seu acionamento (6), e ainda um display touchscreen (7) para interação do usuário com a bancada, o qual permite alterações de parâmetros e apresenta as magnitudes das variáveis relevantes sobre o sistema em funcionamento. Foi disponibilizado à comunidade acadêmica do IFSC, no Apêndice A, os materiais didáticopedagógicos desenvolvidos, como manual de utilização, desenhos desenvolvidos e vídeos de instrução (sem áudio). Assim, nesta seção, serão descritas cada parte do desenvolvimento do protótipo final as quais são unidas de acordo com o diagrama da Erro! Fonte de referência não encontrada.b. 
Figura 1 - a) Componentes do protótipo. b) Diagrama de interconexão.

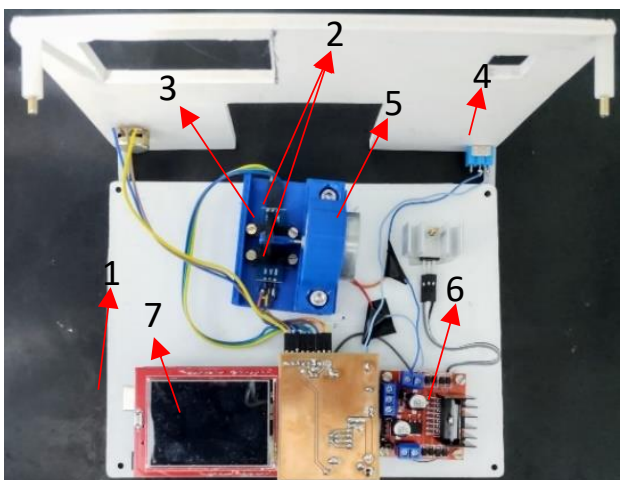

a)

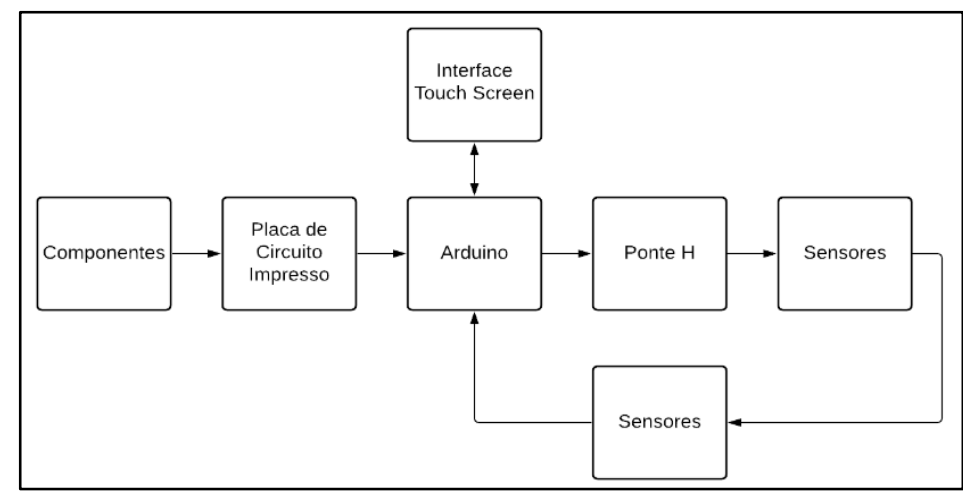

b)

Fonte: Autoria Própria

\section{$2.1 \quad$ Estrutura}

A estrutura da bancada foi construída com o auxílio da manufatura aditiva proveniente da indústria 4.0 que consiste na fabricação de peças a partir de um desenho digital (feito com um software de modelagem tridimensional), por meio de uma impressora 3D disponível na instituição. Dessa forma, o suporte para o motor e o acoplamento entre eixo e disco do encoder, apresentados na Figura 2, foram fabricados em material ABS (Acrylonitrile Butadiene Styrene).

Figura 2 - a) Suporte do Motor. b) Acoplamento para disco do encoder.

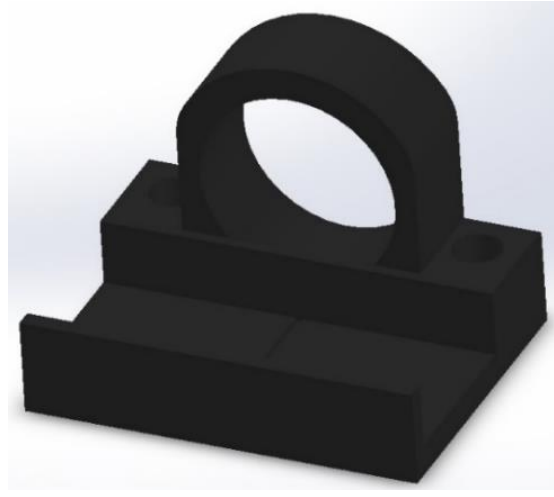

a)

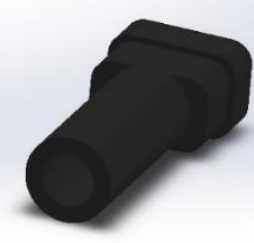

b)

Fonte: Autoria Própria

Além das peças impressas, os componentes fixados em chapas de PVC (polyvinyl chloride) de dimensões $210 \times 150 \mathrm{~mm}$ com um adesivo na parte superior indicando os componentes do sistema. As chapas são fixadas entre si através parafusos em suas extremidades, bem como os componentes presentes nela para que se possa realizar 0 transporte seguro da bancada.

\subsection{Motores e Acionamento}

Cada bancada é constituída de um motor de corrente contínua de $12 \mathrm{~V}$ modelo $6 H 2 L W S K C$, fixado na bancada por meio de parafusos do tipo allen, em uma peça impressa especificamente para esse motor (ver seção 2.1). O acionamento do motor é feito através de uma ponte H L298N usada para prototipagem, que opera entre $4 \mathrm{~V}$ a $35 \mathrm{~V}$ com corrente 
máxima de $2 A$, especificações que suprem seu uso para o motor em questão. O controle é realizado no Arduino (seção 2.4) e o sinal enviado para a ponte através das portas lógicas conforme a Tabela 2.

\subsection{Placa de Circuito Impresso}

A Placa de Circuito Impresso (PCl) também foi feita dentro da instituição, utilizando o software adequado para o projeto e, posteriormente, realizando a corrosão do cobre para realizar a solda dos componentes. A PCl apresentada na Figura 3, é usada para organização dos sinais da bancada e não é realizado nenhum condicionamento de sinal.

Figura 3 - Placa de Circuito Impresso desenvolvida.

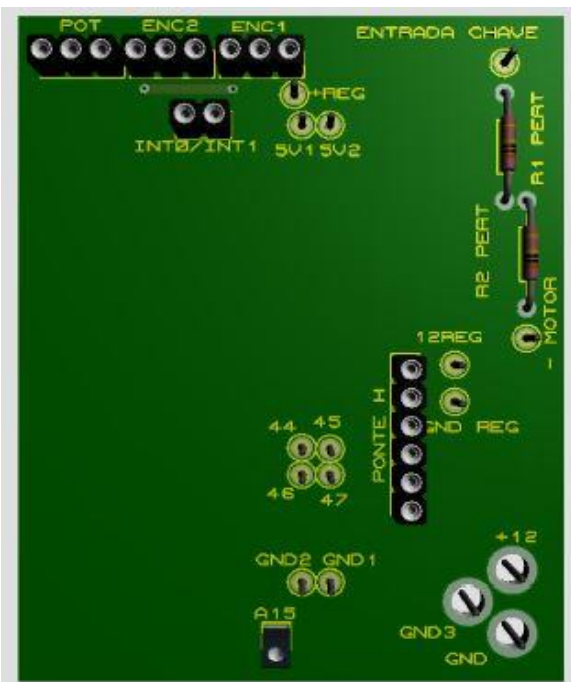

a)

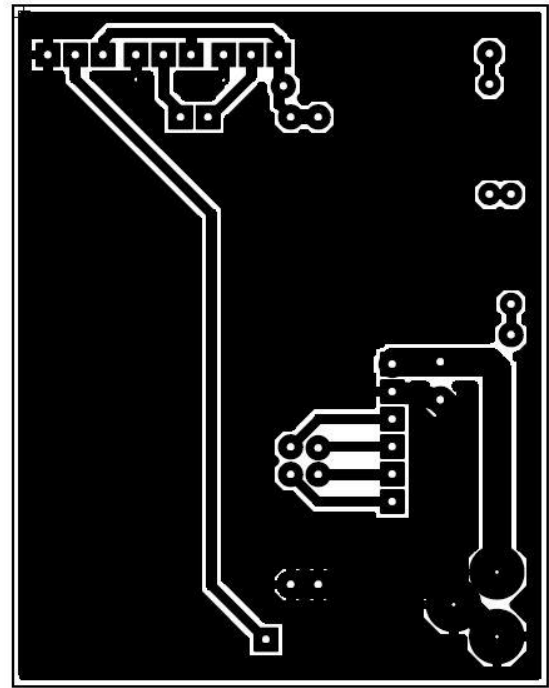

b)

Fonte: Autoria Própria.

A PCl desenvolvida tem suas ligações descritas na Tabela 1 e é acoplada diretamente ao Arduino, semelhante a um shield, no qual recebe e envia os sinais dos componentes da bancada como um todo (seção 2.4).

Tabela 1 - Conexão da PCl com Arduino.

\begin{tabular}{c|c}
\hline PCI & Significado e Conexões \\
\hline POT & $5 V$, GND e A15 \\
\hline ENC2/1 & $5 \mathrm{~V}$, GND e INT0/1 \\
\hline$+5 V 1 / 2$ & PV Arduino $21 / 20$ \\
\hline INT0/1 & $\begin{array}{c}\text { Chave de acionamento da } \\
\text { perturbação }\end{array}$ \\
\hline Entrada Chave & Resistores de perturbação \\
\hline R1/2 Pert & $\begin{array}{c}\text { Saída da perturbação para o } \\
\text { motor }\end{array}$ \\
\hline- Motor & Portas 44 a 47 \\
\hline 44 a 47 & Entrada do regulador de tensão \\
\hline+ REG & GND do regulador de tensão \\
\hline GND REG & Saída para regulador de tensão \\
\hline+12 REG & GND Arduino \\
\hline GND1/2 & GND da fonte externa \\
\hline GND/GND3 & Entrada da fonte externa \\
\hline+12 &
\end{tabular}

Fonte: Autoria Própria. 
A conexão específica entre as portas da ponte $\mathrm{H}$ e Arduino é descrito na Tabela 2.

Tabela 2 - Conexão das portas entre ponte H e Arduino.

\begin{tabular}{c|c}
\hline Porta Arduino & Porta Ponte H \\
\hline+12 & $+12 \mathrm{~V}$ \\
\hline GND3 & GND \\
\hline 44 & $\ln 1$ \\
\hline 45 & $\ln 2$ \\
\hline 46 & $\ln 3$ \\
\hline 47 & $\ln 4$ \\
\hline
\end{tabular}

Fonte: Autoria Própria.

\subsection{Componentes Eletrônicos e Instrumentação}

Toda a eletrônica da bancada foi baseada no funcionamento do microcontrolador Atmega 2560 embarcado na plataforma Arduino Mega. Logo, todos os componentes eletrônicos utilizados foram comprados do que já se tem no mercado, visando a prototipagem em conjunto com a plataforma. $\mathrm{O}$ controle do motor é realizado pela ponte $\mathrm{H}$, que recebe o sinal do Arduino, de acordo com os sinais lidos a partir dos sensores. Os sensores, por sua vez, são dois sensores infravermelhos (modelo H206) funcionando como encoders juntamente com uma espécie de disco perfurado acoplado ao motor, usados para realizar a contagem do número de rotações do motor. Dessa forma, em conjunto com o microcontrolador, é realizada a leitura da velocidade angular do motor sempre que a luz das chaves forem interrompidas pelo disco.

Atendendo um dos requisitos de projeto, que é ter a possibilidade de inserir uma perturbação conhecida no sistema, são usados dois resistores de $10 \Omega$ em série entre si e em paralelo com o motor, acionados por uma chave de duas posições. Com isso, quando a chave é acionada faz com que parte da corrente destinada ao motor seja drenada para os resistores, fazendo com que o controlador regule a tensão enviada ao motor para manter a velocidade igual a referência inserida. Para a inserção da referência, é utilizado um potenciômetro de $5 k \Omega$, como divisor de tensão, em uma porta analógica do microcontrolador.

Para a alimentação da bancada, é usado uma fonte chaveada de $12 \mathrm{~V}$ e $1 \mathrm{~A}$ compatível com os demais componentes. Para a alimentação correta dos sensores de velocidade, é utilizado um regulador de tensão LM7805 que regula a tensão proveniente da fonte. Outro requisito solicitado, é que se tenha algum tipo de comunicação com o usuário e a possibilidade da realização da modelagem matemática do sistema. Assim, foram implementadas duas interfaces sendo uma local, que possibilita o controle e a visualização dos dados, e outra que funciona como um supervisório, ambas descritas na seção 2.6.

\subsection{Algoritmo de Operação e Controle}

O algoritmo foi desenvolvido na IDE (Integrated Development Environment) do próprio Arduino, na linguagem C. A aquisição e tratamento dos sinais dos sensores infravermelhos é feita por meio de uma interrupção externa para cada sensor, tornando-os sinais de um encoder ao passo que alteram seu sinal lógico, fazendo a leitura por quadratura, amplificando a resolução dos sensores. Assim, com a leitura do sinal do motor, é realizado o cálculo da velocidade angular do motor em rad/s para fechar a malha de controle do sistema. O controlador da $C[k]$, por sua vez, é do tipo Proporcional Integral Derivativo (PID), já apresentado em sua forma discreta na Equação (1)

$$
C[k]=K_{p} e[k]+\frac{T_{s}}{K_{i}} e[k-1]+\frac{K_{d}}{T_{s}}(e[k]-e[k-1]),
$$


no qual $K_{p}, K_{i}$ e $K_{d}$ representam os ganhos proporcional, integral e derivativo, respectivamente. O erro é dado por $e(k)$ e $T_{s}$ é o período de amostragem do sistema.

Para a programação da interface local na tela touchscreen, foi utilizada a biblioteca disponibilizada pela IDE. Além da bancada, toda a coleta e tratamento de dados é realizado ao longo do algoritmo bem como a comunicação USB com o supervisório. Para consulta, o código desenvolvido está disponível no Apêndice A.

\subsection{Interfaces}

As interfaces para interação com o usuário foram desenvolvidas em FUZINATTO E MILHOMEM (2018) e adaptadas ao projeto, uma vez que se busca a adequação do hardware para facilitar a construção de um número maior de bancadas e seu uso em aulas da área de engenharia de controle. Assim, para melhor apresentação, as interfaces utilizadas na bancada serão explanadas separadamente, na sequência.

\section{Interface Local}

A interface local permite a visualização dos principais dados, por exemplo, funções, indicador de velocidade e alteração de parâmetros do controle. Ao iniciar a bancada, a tela inicial do sistema é apresentada na Figura 4a, a qual conta com os sinais de referência, velocidade e tensão enviada ao motor na parte superior. Outros dois botões podem ser vistos, usados para inicializar ou realizar ajustes nos parâmetros do controlador conforme Figura 4b. Dessa forma, pode-se utilizar a bancada isoladamente para demonstração e testes do controlador apenas utilizando a fonte externa.

Figura 4 - a) Tela inicial. b) Tela de alteração de parâmetros.

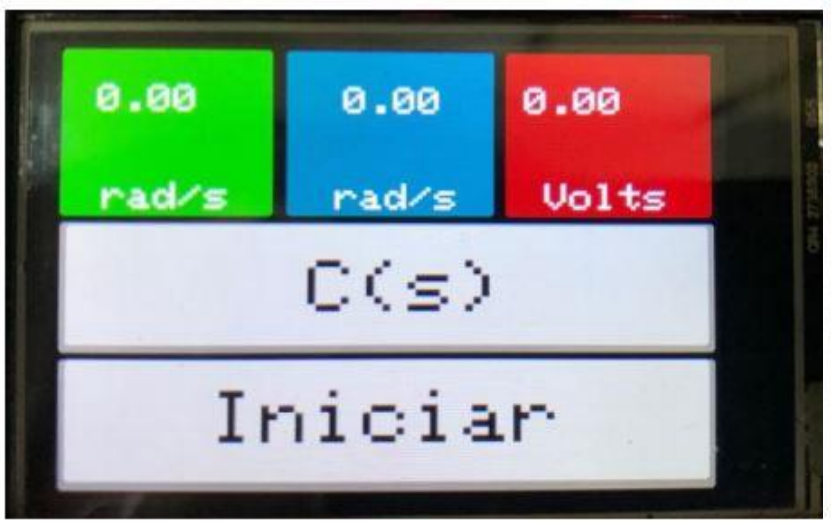

a)

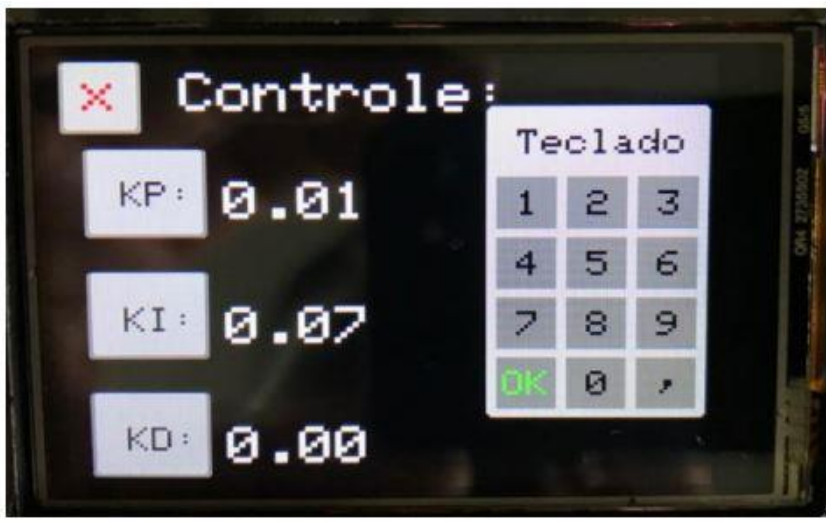

b)

Fonte: Autoria Própria.

\section{Supervisório}

A interface remota funciona também como um sistema supervisório, com mais indicadores e aplicações de diferentes entradas para realizar a modelagem matemática da planta e gráficos com respostas para cada opção de controle. O supervisório foi desenvolvido na linguagem $C \#$ no software Visual Studio e tem como tela inicial a ilustração de como funciona a bancada por completo, apresentando os sinais lidos e enviados.

Para iniciar os testes para obtenção de dados ou mesmo os testes do controlador, é preciso conectar o Arduino em uma das portas USB do computador, assim realizar a conexão do software com a bancada. Feito isso, a tela inicial remete diretamente à realização da modelagem matemática tornando possível testar qualquer sinal de entrada, entre eles degrau, rampa, impulso e o modo manual que funciona a partir de uma definição 
da referência. A tela apresentada na Figura 5, permite a visualização de dois mostradores digitais, um deles a velocidade atual do motor em radianos por segundo $(\mathrm{rad} / \mathrm{s})$, e a tensão aplicada no motor, conforme a bancada esteja funcionando em modo operação.

Logo após o teste, o software gera um arquivo com o vetor de dados da bancada para a realização da modelagem em software específico. Outra funcionalidade presente no software é a tela de controle que, basicamente, propõe o mesmo uso e função da interface local, contando com os ajustes no controlador, modificação dos valores de referência e leitura dos sinais da bancada, conforme a Figura 6 .

Figura 5 - a) Tela inicial do supervisório. b) Tela de controle do supervisório.

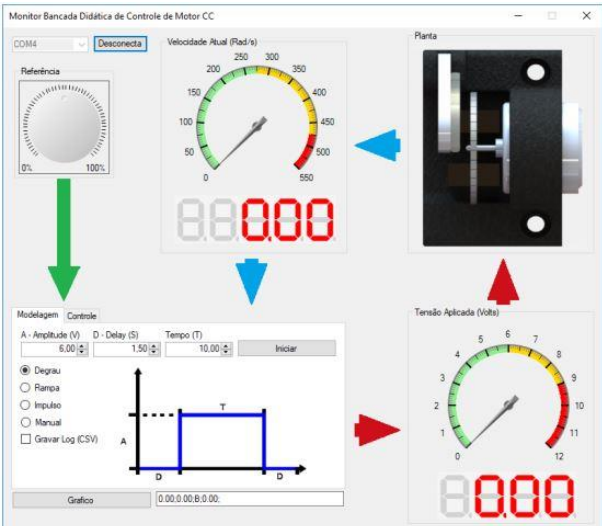

a)

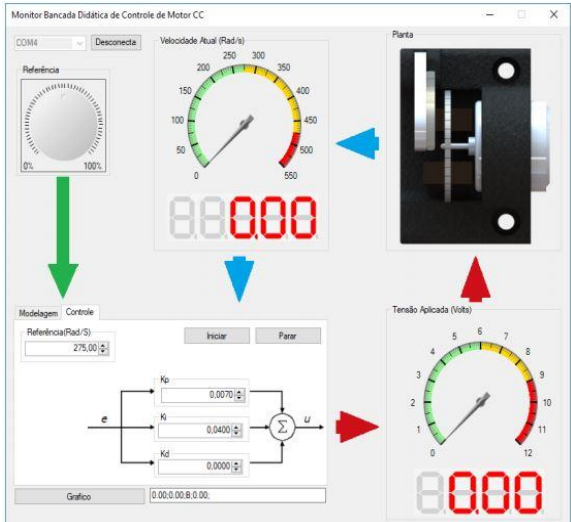

b)

Fonte: Autoria Própria

Além das telas de controles e modelagem, é possível ter acesso ao gráfico gerado instantaneamente em relação aos testes realizados. A Figura 6 mostra um exemplo de teste realizado ilustrando os sinais da bancada, sinal de referência em verde, a tensão em vermelho e a velocidade do motor em azul.

Figura 6 - Teste com entradas do tipo: a) Degrau, b) Rampa e c) Impulso.

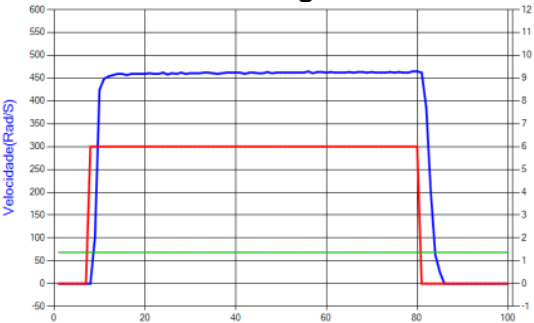

a)

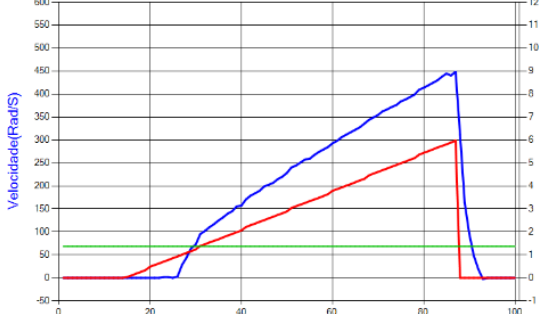

b)

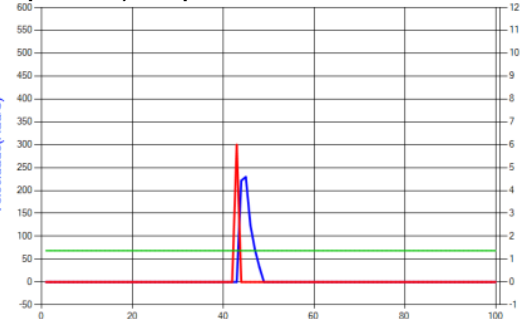

c)

Fonte: Autoria Própria

\section{RESULTADOS}

Serão apresentados nessa seção os resultados encontrados com o desenvolvimento do projeto, buscando apresentar o funcionamento do sistema a partir de uma metodologia desenvolvida para utilização no material didático-pedagógico, para emprego em disciplinas da área de sistemas de controle, buscando a modelagem matemática do processo. Adicionalmente, apresentam-se os resultados pedagógicos observados durante o trabalho, que teve parte do seu desenvolvimento realizado de forma remota, por conta da suspenção das atividades presenciais na instituição. A Figura 7 representa as bancadas construídas 
em seu estado final com a junção de todos os itens apresentados na seção anterior, com o adesivo superior indicando os componentes que a integram e seu funcionamento.

Figura 7 - Bancadas construídas.

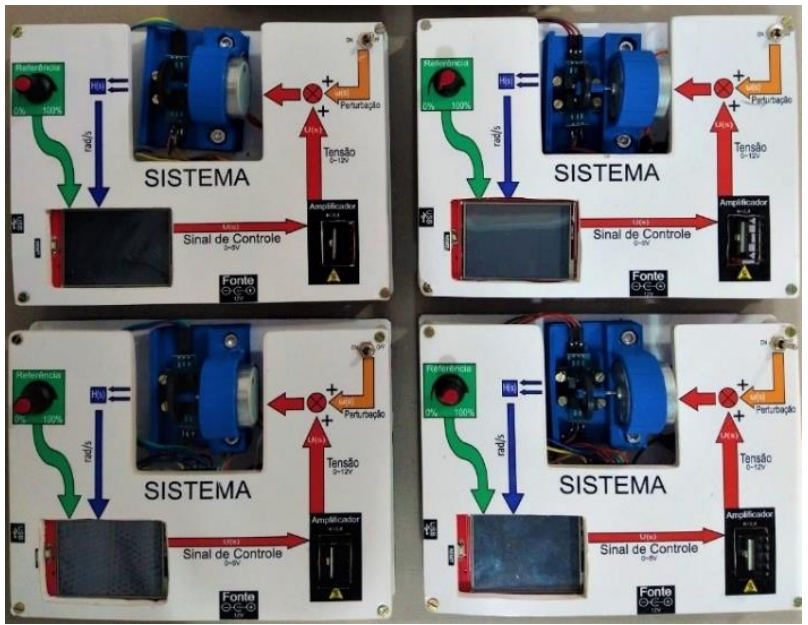

Fonte: Autoria própria.

Durante o desenvolvimento das bancadas, foram adequados alguns itens para auxílio na manutenção do protótipo, como os sensores e o disco do encoder que são amplamente comercializados. Além disso, foi inserido uma perturbação conhecida, apresentando uma melhoria em relação ao projeto de FUZINATTO E MILHOMEM (2018), uma vez que permite aos docentes a exemplificação deste fator, bem como a identificação e controle realizando a rejeição do sinal de perturbação. A partir disso, tem-se a Tabela 3 com os valores médios dos componentes utilizados. Assim, a montagem das bancadas deu-se de forma a obter as ligações como o diagrama da Figura 1b, realizando as conexões para que a comunicação fosse realizada de forma correta entre os componentes. A Tabela 3 apresenta o custo para se produzir uma unidade.

Tabela 3 - Tabela de Custos.

\begin{tabular}{c|c}
\hline Componente & Valor \\
\hline Arduino Mega & $\mathrm{R} \$ 125,87$ \\
\hline Display LCD & $\mathrm{R} \$ 86,76$ \\
\hline Disco do encoder & $\mathrm{R} \$ 9,34$ \\
\hline Motor & $\mathrm{R} \$ 18,90$ \\
\hline Ponte H & $\mathrm{R} \$ 22,71$ \\
\hline Sensor & $\mathrm{R} \$ 20,80$ \\
\hline Regulador de tensão & $\mathrm{R} \$ 2,80$ \\
\hline Dissipador de calor & $\mathrm{R} \$ 3,00$ \\
\hline Potenciômetro & $\mathrm{R} \$ 2,51$ \\
\hline $\begin{array}{c}\text { Resistores de } \\
\text { perturbação }\end{array}$ & $\mathrm{R} \$ 2,70$ \\
\hline $\begin{array}{c}\text { Chave de acionamento } \\
\text { da perturbação }\end{array}$ & $\mathrm{R} \$ 4,50$ \\
\hline Fonte 12V 1A & $\mathrm{R} \$ 19,90$ \\
\hline Cabos e conectores & $\mathrm{R} \$ 33,50$ \\
\hline Parafusos e porcas & $\mathrm{R} \$ 3,00$ \\
\hline Estrutura para fixação & $\mathrm{R} \$ 50,00$ \\
$\begin{array}{c}\text { Adesivo de } \\
\text { identificação }\end{array}$ & $\mathbf{R} \$ 406,29$
\end{tabular}

Fonte: Autoria Própria. 


\subsection{Exemplo de Operação da Bancada: Modelagem do Processo}

Para demonstração de parte dos resultados obtidos, será apresentado a metodologia utilizada para a modelagem e controle do sistema construído, que integra o material didático-pedagógico desenvolvido para instruir os discentes como operar as bancadas da Figura 7. Segue:

- Conecte o cabo USB do Arduino em um computador e a fonte de alimentação;

- Acesse o software supervisório, disponível no Apêndice A, e selecione, na parte superior esquerda, a porta COM na qual está conectado o Arduino;

- Após a conexão e inicialização da bancada, é possível acessar a aba modelagem;

- Para extrair os dados de dinâmica do sistema, podem ser escolhidos três tipos de sinais: degrau, rampa ou impulso. Pode-se modificar os parâmetros dos sinais, como: delay, duração e amplitude. A título de exemplificação, será usado a entrada do tipo degrau com amplitude de $8 \mathrm{~V}$, delay de $2 \mathrm{~s}$ e duração de $10 \mathrm{~s}$ (Figura 8a);

- Depois de selecionar os parâmetros, pressione o botão iniciar e a planta iniciará o algoritmo do sinal escolhido;

- Ao término do teste, o software irá exportar os dados em um arquivo em formato "csv" na sua área de trabalho com o nome 'Modelagem' seguido da data/hora da realização;

- Para a identificação do sistema, é preciso abrir o software Matlab e pressionar o botão Importar Dados (Import Data);

Figura 8 - Exemplo de entrada para modelagem matemática.

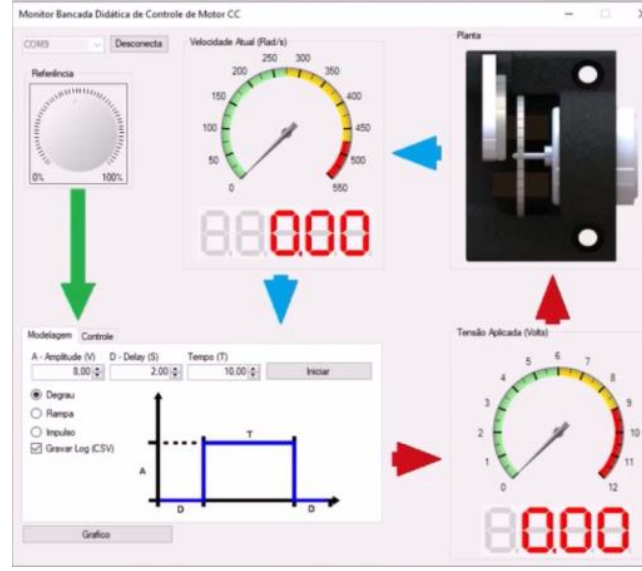

a)

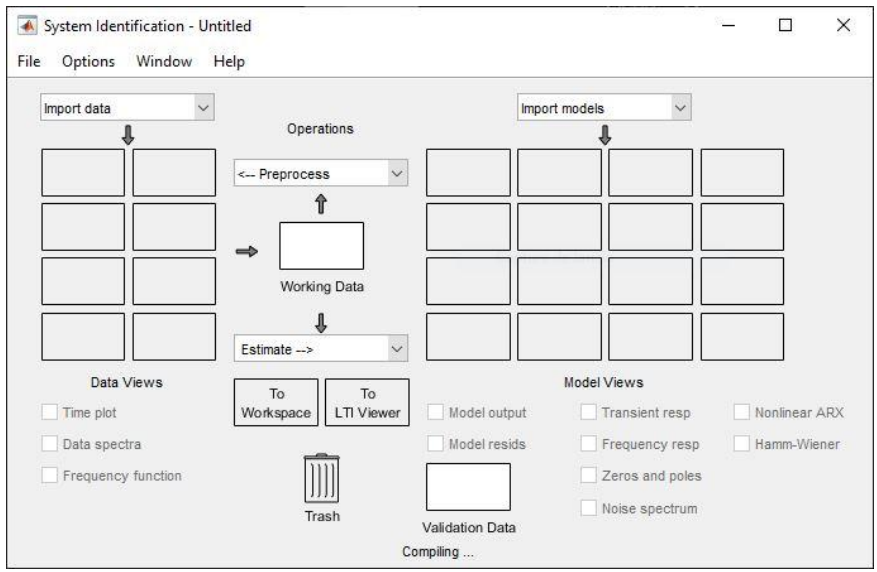

b)

Fonte: Autoria Própria.

- Dentro da janela de importar dados, selecione as opções: Opção Delimitado (Delimited) e Delimitador de colunas ponto e vírgula (Semicolon);

$\checkmark$ Selecione o intervalo de dados que deseja importar (geralmente todas as linhas) e pressione o botão importar seleção (Import Selection);

$\checkmark$ Digite na tela de workspace o comando 'ident' para abrir a tela igual a Figura 8b;

$\checkmark$ Dentro do 'ident', no menu importar dados (Import Data), selecione importar dados no domínio do tempo (import time domain data);

$\checkmark \quad$ No campo entrada (input), selecione ou digite o nome da variável da primeira coluna dos dados importados;

$\checkmark$ No campo saída (output), selecione ou digite o nome da variável da segunda coluna dos dados importados; 
$\checkmark$ Pressione o botão 'import';

$\checkmark$ No menu estimar (Estimate), selecione a função de transferência do modelo (Transfer Function Model);

$\checkmark$ Arraste a função gerada até a caixa para espaço de trabalho (To Workspace);

$\checkmark$ A função de transferência estimada estará na seção das variáveis do Matlab.

Com a identificação do modelo realizada, o usuário está apto a desenvolver o projeto do controlador. No exemplo mencionado, o modelo é apresentado na Equação (2).

$$
G(s)=\frac{-7.88 s+157.6}{s^{2}+4.215 s+3.428}
$$

Fazendo uso da metodologia mencionada, o discente poderá realizar o projeto do controlador que deseja e aplicá-lo na bancada e, a partir disso, observar o comportamento do sistema, baseado nos conceitos teóricos apresentados em aula. Após a obtenção da função de transferência (2), usa-se a lei de controle (1), a partir dos ganhos $K_{p}=0.01, K_{i}=$ 0.07 e $K_{d}=0$. Com isso, a

Figura 9a apresenta de forma simplificada a malha de controle utilizada no sistema e, na Figura $9 \mathrm{~b}$ tem-se a representação de um teste realizado com a bancada, apresentando a aplicação de um degrau de referência de $275 \mathrm{rad} / \mathrm{s}$, e, em regime permanente, 0 seguimento da referência aplicada.

Figura 9 - a) Malha de controle simplificada. b) Gráfico gerado pelo software.

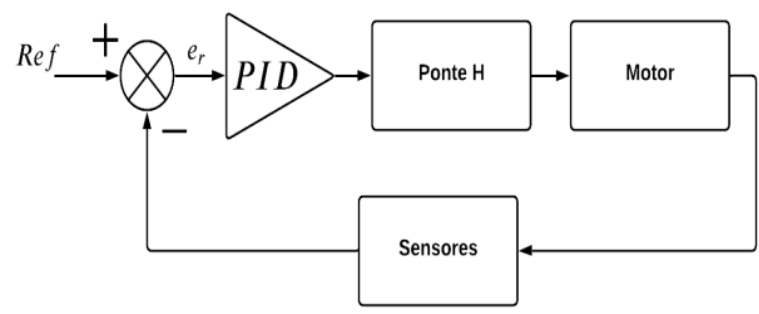

a)

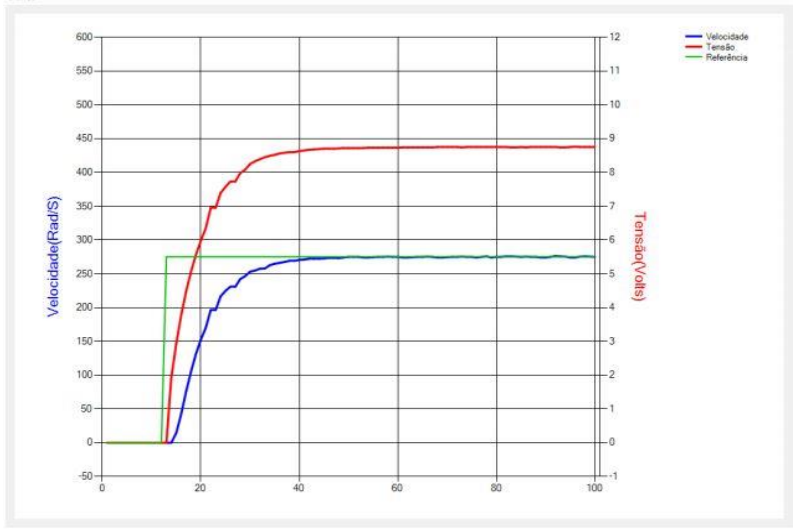

b)

Fonte: Autoria Própria.

No Apêndice A é possível observar alguns vídeos de demonstração desenvolvidos (sem áudio), apresentando o funcionamento da bancada com: a interface local; o supervisório; a visualização dos gráficos; a apresentação da metodologia descrita nessa seção. Com isso, no que diz respeito a modelagem matemática e simulação do sistema, obteve-se um resultado satisfatório, uma vez que o software gera um arquivo com todos os dados para a identificação do sistema, o que tornar possível o projeto do controlador para o protótipo, a partir de projeto de controladores clássicos e posteriormente seu emprego na bancada a partir da Equação (1). 


\subsection{Resultados Pedagógicos observados durante as atividades remotas}

Devido ao cenário de pandemia, algumas etapas do desenvolvimento dos protótipos/bancadas foram alteradas em função da suspensão de atividades presenciais no IFSC, Campus Chapecó. Atividades relacionados a testes práticos em laboratórios foram alterados para simulações via software, buscando encontrar um funcionamento próximo ao desejado para a bancada. Ainda assim, algumas etapas de fabricação relacionadas a montagem final e testes das bancadas foram desenvolvidas pelo estudante produtor, porém fora dos ambientes dos laboratórios da instituição, o que trouxe um desafio maior ao desenvolvimento do projeto do sistema. Outro fator que auxiliou o desenvolvimento do projeto de forma remota, se deve a disponibilidade de um computador para acesso remoto com os softwares necessários a simulação e desenvolvimento dos projetos das peças para fabricação na impressora 3D.

Nesse sentido, as atividades desenvolvidas foram de grande importância a formação do estudante produtor pois enfatizaram e aprimoraram as capacidades de trabalho com relação às competências técnicas as quais a bancada/protótipo se aplica (como: programação, instrumentação e sistemas de controle) e competências comportamentais, como resiliência e adaptação, devido a adequação a mudança do ambiente de trabalho e das condições para desenvolvimento das atividades (realizadas de forma remota).

\section{CONCLUSÃO}

A utilização de protótipos como ferramentas e métodos didáticos facilitam o processo de ensino-aprendizagem, tornando o ensino em engenharia mais atrativo a partir de uma abordagem prática e contextualizada, fomentando a busca por informação e reforçando a necessidade de integrar conhecimentos. Assim, o uso de equipamentos técnicos, então, de maneira condizente com as ementas a serem apresentadas pelo docente, permite aos estudantes a obtenção de experiência através de implementações repetitivas e análise de resultados em sistemas similares aos encontrados no mercado de trabalho, considerando o custo, a manutenção, montagem e portabilidade do sistema. Desta forma, os objetivos do trabalho foram alcançados de forma satisfatória, uma vez que foi desenvolvido e adaptado um sistema capaz de auxiliar docentes e discentes em atividades de ensino-aprendizagem voltadas a modelagem matemática e controle de um sistema dinâmico, além da produção de um material didático-pedagógico disponível no Apêndice $A$.

No cenário de pandemia, em que as atividades presenciais de ensino e pesquisa foram suspensas, algumas etapas do trabalho foram alteradas. Assim, atividades práticas de realizadas em laboratórios do IFSC Campus Chapecó foram alteradas para simulações via software, e uso de equipamentos de forma remota. Apesar disso, as atividades desenvolvidas conseguiram atingir seus objetivos, produzindo as peças necessárias para montagem das bancadas, através de desenhos auxiliados por computador, impressão 3D, e o auxílio de docentes e técnicos que tinham acesso a infraestrutura do Campus.

Os benefícios pedagógicos alcançados foram observados apenas com relação as contribuições para a formação do estudante produtor do trabalho, que pode desenvolver competências técnicas e comportamentais durante o projeto. Contudo, em um próximo trabalho, estima-se investigar sobre as contribuições pedagógicas que a ferramenta de ensino possa propiciar no ensino presencial. Mais adiante, sugere-se o desenvolvimento de trabalhos que foquem na construção de experimentos padrões de laboratório para o sistema apresentado, além do desenvolvimento de simuladores que possam emular 0 sistema e serem usados de forma remota, auxiliando no processo de ensino-aprendizagem em engenharia, atividades de pesquisa e extracurriculares. 


\title{
Agradecimentos
}

Ao Instituto Federal de Educação, Ciência e Tecnologia de Santa Catarina (IFSC) Campus Chapecó, pelo financiamento do projeto, e ao LABICON (Laboratório de Instrumentação e Controle) pelo uso da infraestrutura, de forma remota.

\author{
Apêndices \\ Apêndice A: https://tinyurl.com/899vhuth .
}

\section{REFERÊNCIAS}

ÁLVARES, Vanessa Oliveira de Moura. O Docente-Engenheiro Frente Aos Desafios Da Formação Pedagógica No Ensino Superior. 2006. Dissertação (Mestrado) - Curso de Pós-Graduação em Educação da Universidade Federal de Uberlândia - UFU, Uberlândia, 2006. Disponível em: https://tinyurl.com/5xxp8k5f. Acesso em: 07 abr. 2021.

BENDER, W. N. Aprendizagem baseada em projetos, educação diferenciada para o século XXI. 1. ed. São Paulo: PENSO Editora, 2014.

FIORE FERRARI, Eduardo; LEYMONIE SAENZ, Julia. Didáctica práctica: para enseñanza básica, média y superior. Modevideo: Grupo Magro, 2018.

FUZINATTO, Arthur; MILHOMEM, Rômulo Lira. Bancada didática de Controle De Motor CC. In: Seminário de Ensino, Pesquisa e Inovação do IFSC, 2018. Florianópolis. Anais. Disponível em: https://tinyurl.com/6678y3bj . Acesso em 07 abr. 2021.

OLIVEIRA, Vanderli Fava de; TOZZI, Marcos José; LODER, Liane Ludwig. Desafios da Educação em Engenharia: Formação em Engenharia, Capacitação Docente, Experiências Metodológicas e Proposições. Brasília: ABENGE, 2014.

ROZENFELD, Henrique et al. Gestão de Desenvolvimento de Produtos - Uma Referência para a Melhoria do Processo. São Paulo: Saraiva. 2006.

\section{PEDAGOGICAL EXPERIENCES IN THE DEVELOPMENT AND DIDATIC ADEQUACY OF PROTOTIPES FOR MULTIDISCIPLINARY PRACTICES IN ENGINEERING COURSES}

\begin{abstract}
The development of didactic prototypes is a consolidated teaching-learning practice in the sense of contributing to the formation of the students involved, since it is a tool that favors the integration between theory and practice, which points to a formative process focused on knowing how to do, introducing the students involved to the world of work in which they will be inserted. Thus, this paper illustrates an experience of the Campus Chapeco, of the Instituto Federal de Santa Catarina, in the development and adaptation of teaching prototypes for multidisciplinary practices in the Control and Automation Engineering course. The theoretical and practical results and their impacts on research and teaching of the academic community in which it is inserted are reported, from the perspective of teachers and students.
\end{abstract}

Keywords: Didactic Prototypes, Control and Automation Engineering, Engineering Education. 